\title{
O PROBLEMA DOS PÓRTICOS: \\ UMA INTERVENÇÃO DIDÁTICA CONSTRUÍDA PARA A DISCIPLINA DE CÁLCULO DIFERENCIAL INTEGRAL
}

Gabriel Loureiro de Lima-gllima@ pucsp.br

Barbara Lutaif Bianchini - barbara@ pucsp.br

Pontifícia Universidade Católica de São Paulo - PUC-SP

Rua Marquês de Paranaguá, 111

01303-050 - São Paulo - SP

EloizaGomes-eloiza@maua.br

Instituto Mauá de Tecnologia - IMT

Praça Mauá, 1

09580-900 - São Caetano do Sul - SP

Simone Leal Schwertl-silealschwertl@gmail.com

Fundação Universidade Regional de Blumenau - FURB

Rua Antônio da Veiga, 140

89012-570 - Blumenau - SC

Resumo: $O$ objetivo deste artigo, um dos frutos das demandas provenientes de reflexões ocorridas nos encontros de 2017 a 2019 do Grupo de Trabalho Ciências Básicas e Matemática na Engenharia, vinculado à Associação Brasileira de Educação em Engenharia, é apresentar uma intervenção didática, desenvolvida, para uma disciplina inicial de Cálculo Diferencial e Integral, a partir de um problema clássico da Engenharia Civil - o problema dos pórticos - elaborado por meio do diálogo entre professores da área de Matemática e docentes que ministram disciplinas específicas ou profissionalizantes nos cursos de Engenharia da habilitação mencionada. Explicitamos o problema que contextualiza a intervenção proposta, planejada para ser desenvolvida em 12 horas/aula (aproximadamente 3 semanas com 4 horas/aula em cada uma delas), e, em seguida, detalhamos o que foi concebido para ser desenvolvido em cada uma das aulas contemplando tal intervenção. Entendemos que a proposta apresentada tem potencial para, na formação do futuro engenheiro, proporcionar o desenvolvimento ou a mobilização tanto de algumas competências gerais explicitadas nas novas Diretrizes Curriculares Nacionais para os cursos de Graduação em Engenharia, quanto de competências específicas da área da Matemática postuladas por Mogens Niss.

Palavras-chave: Engenharia Civil. Problema dos Pórticos. Cálculo Diferencial e Integral. Intervenção Didática. Competências.

\section{INTRODUÇÃO}

Esse artigo é um dos frutos das demandas surgidas a partir das discussões efetivadas no âmbito do Grupo de Trabalho Ciências Básicas e Matemática na Engenharia (GT-CbME), vinculado à Associação Brasileira de Educação em Engenharia (ABENGE), realizadas entre 2017 e 2019. Nesse período o GT teve como objetivo promover reflexões sobre o ensino das Ciências Básicas e Matemática $(\mathrm{CbM})$ nos cursos de Engenharia, tendo como foco principal - 
na perspectiva de qualificar os processos de ensino e de aprendizagem - o diálogo entre os professores das áreas específicas e aqueles que ministram as disciplinas de CbM. Para os cinco primeiros anos de atuação, o GT-CbME teve entre as suas metas o levantamento dos desafios atuais para qualificar o ensino das $\mathrm{CbM}$, tendo em vista, especialmente, as novas Diretrizes Curriculares Nacionais para os cursos de Engenharia (DCN), homologadas em 2019.

Dentre os desafios apontados nas discussões conduzidas pelos membros do GT ficou latente a questão da falta de material didático contextualizado para as disciplinas de CbM, elaborados a partir de problemas fortemente relacionado às diversas áreas da Engenharia. Esta preocupação tem no seu cerne a qualificação da Educação em Engenharia e a motivação dos estudantes, questões consonantes com as orientações das novas DCN, tanto no contexto do acolhimento dos estudantes, quanto na possibilidade de mobilização/desenvolvimento de competências gerais almejadas para o egresso da Engenharia.

No presente texto focaremos principalmente em competências propostas pelas novas DCN fortemente relacionadas à área da Matemática. Nesse sentido, é importante destacar que foi consenso nas discussões proporcionadas pelo GT-CbME o entendimento apontado por Lima (2015, p. 6) que "a contextualização de um conceito matemático pode ser relacionada ao processo de construção de significados do referido conceito". No caso específico das engenharias, a contextualização está relacionada à ideia de vincular os conceitos matemáticos a questões referentes às disciplinas específicas e profissionalizantes a serem cursadas pelos estudantes, quanto às situações que estes enfrentarão em suas futuras vidas profissionais (BIANCHINI et al., 2017).

Sendo assim, compreendemos e defendemos, com o aval de nossas pesquisas na área de ensino na Engenharia, de nossa experiência como docentes da área da Matemática, e pelas discussões desencadeadas no GT-CbME entre 2017 e 2019, que a abordagem de problemas contextualizado nas disciplinas básicas a partir de problemas das áreas específicas e profissionalizantes em cursos de Engenharia, possui potencial significativo para mobilizar as competências gerais elencadas pelas novas DCN (BRASIL, 2019). De outra parte, no caso específico da Matemática, a contextualização supracitada, viabiliza a construção de significados dos conceitos matemáticos em consonância com a mobilização de competências específicas da área. A seguir será apresentada uma intervenção didática, com forte potencial para mobilização de competências, construída a partir de um problema que emergiu do diálogo entre professores da área da Matemática (os três primeiros autores deste artigo) e professores engenheiros que ministram disciplinas específicas ou da área profissionalizante em um curso de Engenharia Civil.

\section{A INTERVENÇÃO DIDÁTICA}

A intervenção didática foi desenvolvida para ser aplicada em 12 horas/aula (aproximadamente 3 semanas com 4 horas/aula em cada uma delas, ou seja, $2 \mathrm{~h}$ por aula). No Quadro1 apresentamos o problema que contextualiza a intervenção proposta. Na sequência, explicitamos o detalhamento proposto para cada uma das semanas e suas respectivas aulas.

Quadro 1- O problema dos pórticos

Considere um pórtico com massa $m=384 \mathrm{~kg}$, rigidez de cada pilar $\frac{k}{2}=19200 \mathrm{~N} / \mathrm{m}$ e taxa de amortecimento $\xi=\frac{c}{2 m \sqrt{\frac{k}{m}}}=0,05$, sendo $c$ a constante de amortecimento. Uma força estática é aplicada sobre essa estrutura, causando um deslocamento inicial $u_{0}=0,1 \mathrm{~m}$. Em seguida, essa força é retirada bruscamente e a estrutura passa a vibrar livremente com 
velocidade inicial nula. Considerando apenas a possibilidade de deslocamento horizontal desse pórtico, a expressão que permite analisar o comportamento do deslocamento $u$ em relação ao tempo é dada por:

$$
u(t)=0,10 e^{-0,5 t}[\cos (10 t-0,05)]
$$

Questão: A amplitude do movimento será $20 \%$ da amplitude inicial após quantos ciclos?

Observação: considerar duas casas decimais para os cálculos.

Fonte: elaborado pelos autores

\subsection{Primeira Semana}

\section{$1^{a}$ aula}

Nesta aula, em primeiro lugar, haverá a proposição do problema, disponibilização dos materiais para preparação prévia dos estudantes para uma imersão na temática e para a realização de pesquisas livres relacionadas ao contexto do problema. Em relação aos materiais de preparação prévia, foram produzimos, por engenheiras civis e por físicos, que atuam de forma colaborativa conosco, dois vídeos ${ }^{1}$. Nestes, as principais ideias relacionadas aos pórticos são apresentadas em uma linguagem acessível a estudantes ingressantes, além de haver uma retomada do modelo massa-mola já estudado pelos alunos no Ensino Médio, relacionando-o ao contexto de análise dinâmica de estruturas e, especialmente, aos pórticos. Também como material de apoio, sugerimos a produção de um texto, por engenheiros ou físicos a respeito das noções de amortecimento, taxa de amortecimento, frequência natural, frequência natural amortecida de uma estrutura e ângulo de fase de movimento harmônico.

$\mathrm{Na}$ finalização da aula, os estudantes, organizados em grupos, responderão à Questão 1: A relação $u$ presente no enunciado do problema remete a algum(ns) conceito(s) matemático(s) que você já estudou? Qual(is)?

O objetivo ao propor esta questão é realizar uma sondagem para sabermos a quais conceitos matemáticos o estudante será remetido ao analisar a expressão algébrica de $u$. Esperamos que ele faça referência às ideias de função e, especialmente de funções trigonométricas, exponenciais e também ao produto de funções. Há possibilidade de os alunos fazerem referências ainda às ideias de funções constantes e de composição de funções. $\mathrm{O}$ professor deve sempre estar atento para explorar, no momento mais apropriado da sequência de aulas que estão sendo proposta, estes e outros conceitos que emergirem.

Após os estudantes responderem à questão 1 e conjuntamente a classe perceber que a relação entre o deslocamento horizontal $u$ do pórtico e o tempo $t$ é uma função, o professor deverá solicitar a seguinte tarefa: Tarefa 1: Pesquisar e trazer para a próxima aula uma outra situação (de seu interesse, do cotidiano, da Física, da Engenharia, etc.) que seja modelada por uma função real de uma variável real. A tarefa dessa primeira aula visa possibilitar que as atividades a serem realizadas pelos estudantes na aula seguinte sejam desenvolvidas tendo por contexto situações que eles próprios identificaram e que, portanto, possam estar mais próximas de seus interesses.

\section{$2^{a}$ aula}

O professor, a partir de uma situação escolhida por ele que também seja modelada por uma função real de uma variável real, inicia fazendo uma retomada de aspectos principais relacionados à noção de função: o que caracteriza uma relação funcional, o fato de nem

\footnotetext{
${ }^{1}$ https://youtu.be/cLBb6z5VkyY; $\underline{\text { https://youtu.be/I7R 4zCoa2I }}$
} 
sempre ser possível obter uma representação algébrica para a lei que caracteriza a relação funcional, as noções de domínio, imagem e contradomínio, as diferentes representações para uma relação funcional (algébrica, gráfica, tabular, língua natural). Deve também enfatizar aspectos relacionados ao contexto do problema (por exemplo, do ponto de vista matemático, a relação funcional com a qual ele está trabalhando pode ter como domínio o conjunto dos números reais, mas e no contexto do problema? Qual o subconjunto do domínio que deve ser considerado?).

Finalizada essa breve intervenção do professor, os estudantes novamente deverão reunir-se em grupos e identificar, a partir dos exemplos por eles trazidos para a aula, se as funções consideradas possuem leis que podem ser representadas por uma expressão algébrica, quais seus domínios, contradomínios, imagens, que subconjuntos destes conjuntos devem ser considerados levando em conta o contexto da situação que está sendo modelada. Devem ainda obter as diferentes representações das funções: língua natural, tabular, algébrica e, com o auxílio de softwares, as representações gráficas. Em seguida, responderão a outras duas questões mais uma vez relacionadas à função $u$ do problema do pórtico: Questão 2: Quais são os conjuntos domínio, contradomínio e imagem da função $u$, considerando exclusivamente o contexto matemático? E se considerarmos o contexto do problema? Questão 3: Com o auxílio de um software, represente graficamente a função $u$ e a partir dessa representação gráfica confirme o que você observou na questão 2 .

As questões 2 e 3 têm por objetivo possibilitar que o estudante trabalhe, no contexto do problema proposto, com os conceitos já estudados na Educação Básica e que foram retomados pelo professor no início desta aula.

Após discussões a respeito das atividades realizadas pelos grupos, o professor deverá propor a tarefa 2 para a aula seguinte, a qual tem como objetivo possibilitar ao aluno que observe que, para chegar ao modelo do deslocamento horizontal do pórtico em relação ao tempo, são necessários uma série de conhecimentos matemáticos e físicos com os quais ele irá trabalhar nas disciplinas destas áreas de conhecimentos, normalmente inseridas nos ciclos básicos dos cursos de Engenharia. Tarefa 2: Pesquisar como se chegou à função que modela o deslocamento horizontal do pórtico em relação ao tempo.

\subsection{Segunda Semana}

\section{$1^{a}$ aula}

Em primeiro lugar, o professor deverá retomar a tarefa 2 discutindo com os estudantes que, obviamente, o objetivo ao pedir que pesquisassem como a expressão que modela o deslocamento horizontal do pórtico em relação ao tempo foi obtida, não era que compreendessem todo o processo envolvido na modelagem desta situação. $\mathrm{O}$ que deveria se tornar evidente é o quanto conceitos matemáticos e físicos são importantes para a modelagem e para a resolução de problemas da Engenharia. Esses conceitos serão todos estudados em disciplinas básicas que, portanto, revelam-se como fundamentais para a formação do futuro engenheiro. Finalizada essa discussão, o professor deverá propor que os estudantes respondam à seguinte questão relacionada a função $u$ do problema que está guiando a abordagem proposta:

Questão 4: Na expressão algébrica de $u$, você identifica a presença de funções elementares? Quais?

O objetivo desta questão é iniciar um trabalho de retomada do estudo das funções elementares a partir de uma percepção inicial dos próprios estudantes, tomando por base a função $u$ presente no problema do pórtico. A partir das respostas dadas por eles, o docente abordará, não da maneira como estes conteúdos já foram estudados no Ensino Médio, mas já 
direcionados aos seus empregos na Engenharia e por meio de uma abordagem computacional, a ideia de funções elementares (nesta aula especificamente as constantes, polinomiais, racionais, modulares, definidas por várias sentenças e logarítmicas), operações (adição, produto e composição) e transformações (translação, reflexão, expansão e contração) com esses tipos de funções.

Após essa discussão, deverá ser deixada a Tarefa 3, cujo o objetivo é auxiliar principalmente àqueles estudantes que tiveram pouco ou nenhum contato com o estudo de funções elementares no Ensino Médio. Em certo sentido, os exercícios propostos para a assimilação daquilo que foi trabalhado em sala de aula poderão contribuir para que os estudantes consigam acompanhar de forma mais efetiva o curso que será desenvolvido, uma vez que terão podido recuperar alguns dos conhecimentos prévios necessários. Tarefa 3: Exercícios preparados pelo professor (sempre que possível contemplando situações contextualizadas, mas não excluindo a possibilidade de questões trabalhando somente com o contexto matemático) para que os estudantes possam revisar e reforçar conhecimentos acerca das seguintes funções elementares: constante, polinomiais, racionais, modulares, definidas por várias sentenças e logarítmicas, suas operações e transformações.

\section{$2^{a}$ aula}

Em primeiro lugar, o professor irá propor a seguinte questão: Questão 5: A função $u$ foi obtida a partir de operações e/ou transformações envolvendo funções elementares. Identifique essas funções, operações e/ou transformações. Essa questão tem por objetivo desencadear, a partir da função $u$ presente no problema do pórtico, a abordagem de dois tipos de funções elementares, suas operações e transformações não trabalhadas na aula anterior, a saber: funções exponenciais e trigonométricas.

Finalizada as discussões relacionadas à questão 5 , o professor deverá propor a seguinte tarefa 4 cujo objetivo é idêntico ao da tarefa 3, mas agora considerando as funções exponenciais e trigonométricas. Tarefa 4: Exercícios preparados pelo professor (sempre que possível contemplando situações contextualizadas, mas não excluindo a possibilidade de questões trabalhando somente com o contexto Matemático) para que os estudantes possam revisar e reforçar conhecimentos acerca das seguintes funções elementares: exponenciais e trigonométricas, suas operações e transformações.

\subsection{Terceira Semana}

O objetivo desta terceira semana, como conclusão do trabalho de retomada de conceitos relacionados às funções elementares e oportunidade de os estudantes colocarem em ação todos os conhecimentos construídos, é responder à questão inicial proposta: obter partir da função $u$ cuja expressão algébrica é $u(t)=0,10 e^{-0,5 t}[\cos (10 t-0,05)]$, após quantos ciclos a amplitude do movimento horizontal do pórtico será $20 \%$ da amplitude inicial. Para auxiliar os estudantes na obtenção dessa resposta, uma série de questões auxiliares deverá ser proposta pelo docente nas duas aulas desta semana, como explicitado a seguir.

\section{$1^{a}$ aula}

Nesta primeira aula da semana, o professor deverá propor que, mais uma vez organizados em grupos, os estudantes trabalhem com as seguintes questões:

Questão 6: Representem, no mesmo sistema cartesiano, graficamente as funções cujas expressões algébricas são dadas por $f(t)=0,10 e^{-0,50 t}$ e $g(t)=\cos (10 t-0,05)$. 
Questão 7: Qual seria a resposta do problema caso a função $u$ não fosse a do enunciado do problema, mas $\operatorname{sim} u(t)=g(t)=\cos (10 t-0,05)$ ?

Questão 8: Como vocês poderiam obter a expressão algébrica da função $u$ a partir das funções $f$ e $g$ ?

Questão 9: A partir do que vocês observaram na questão 8, faz sentido a expressão que modela o comportamento do deslocamento $u$ em função do tempo ser dada por: $u(t)=e^{-0,50 t}[0,10 \cos (10 t-0,05)]$ ? Justifique sua resposta.

A questão 6 tem diferentes objetivos: em primeiro lugar, retomar, com o apoio das Tecnologias Digitais de Informação e Comunicação (TDIC), as discussões acerca das funções exponenciais e trigonométricas. Neste sentido os estudantes poderão mobilizar conhecimentos relacionados aos seguintes aspectos: representações algébricas, representações gráficas, domínio, imagem, zeros das funções, período e amplitude de funções trigonométricas, representações gráficas de funções associadas às funções elementares $p(x)=e^{x} \mathrm{e}$ $q(x)=\cos (x)$. Um segundo objetivo é a obtenção da representação gráfica da função $g$ para que o estudante possa ter subsídios para responder à questão 7. Além disso, entendemos que ao analisar a representação gráfica de $g$, possivelmente poderá começar a perceber a necessidade de um fator de amortecimento (que, posteriormente, ele irá compreender que está atrelado ao papel da função exponencial $f$ na expressão algébrica de $u$ ).

O objetivo da questão 7 é evidenciar ao estudante que se a função $u$ fosse de fato uma função trigonométrica, o movimento horizontal do pórtico jamais cessaria, ou seja, o estudante poderá, perceber que a função $u$ que descreve esse movimento não pode ser periódica. Em relação à questão 8 , seu principal objetivo é o de retomar, de maneira vinculada ao contexto do problema, do ponto vista gráfico, o produto de funções. O estudante poderá, por meio desta questão, perceber que a função $u$ é o produto das funções $f$ e $g$ e deverá, analisando as representações destas duas funções, produzir um esboço da representação gráfica da função produto $(u)$. Na questão 9 , o objetivo é que os estudantes percebam, a partir da representação gráfica que construíram na questão 8 , que, ao contrário do que aconteceria se $u=g$, sendo $u$ o produto de $f$ por $g$, conforme os valores de $t$ crescem, os valores de $u(t)$ se tornam muito próximos de zero, ou seja, a movimentação horizontal do pórtico tende a cessar.

Finalizadas as discussões relacionadas às produções dos grupos acerca destas quatro questões, o professor deverá propor a tarefa 5 a qual tem por objetivo, por meio da pesquisa que realizarão, possibilitar aos estudantes que revisitem ou construam conhecimentos que serão essenciais para as questões a serem trabalhadas na aula seguinte para a efetiva solução do problema e conclusão desta abordagem de noções relacionadas às funções por nós proposta por meio da sequência de atividades apresentada. Tarefa 5: Pesquisar a respeito das aplicações de funções periódicas na Física e na Engenharia e também acerca das noções de pseudoperíodo e amplitude e suas aplicações nas áreas mencionadas.

\section{$2^{a}$ aula}

As questões a serem trabalhadas nesta segunda aula da terceira semana são as seguintes:

Questão 10: Utilizando o GeoGebra, representem graficamente as funções $u$ e $g$ em um mesmo sistema cartesiano. Comparem as representações gráficas de $u$ e de $g$, buscando similaridades e diferenças entre os comportamentos de tais funções.

Questão 11: Qual o comportamento de $u$ quando os valores de $t$ tendem ao infinito?

Questão 12: Construa, com o auxílio do GeoGebra, em um mesmo sistema cartesiano, as representações gráficas de $u, f$ e $-f$. Existem valores de $t$ para os quais $u(t)=f(t)$ ? $\quad$ E valores de $t$ para os quais $u(t)=-f(t)$ ? 
Questão 13: Qual o significado, no contexto do problema, para $u(t)<0, u(t)=0$ e $u(t)>0$ ?

Questão 14: Como identificar os ciclos e as amplitudes da função $u$ a partir de sua representação gráfica?

Questão 15: Respondam à questão: A amplitude do movimento será $20 \%$ da amplitude inicial após quantos ciclos?

Pretendemos, por meio da questão 10, que o estudante retome a representação gráfica da função $u$ construída na questão 3 e então, representando no mesmo sistema cartesiano também a função $g$, possa observar semelhanças e diferenças entre tais funções. Neste sentido, destacamos: os máximos e mínimos locais ocorrem para os mesmos valores de $t$; os zeros coincidem; a amplitude da função $u$ não é constante, ao contrário da amplitude de $g$; a função $g$ é periódica, enquanto a função $u$ pode ser classificada como pseudoperiódica, característica que poderá ser melhor explorada na questão 14.

Ao propor a questão 11, temos dois objetivos. O primeiro é reforçar ao estudante que, de fato, $u$ é um modelo matemático adequado para o movimento horizontal do pórtico, uma vez que, quando os valores de $t$ tendem ao infinito, os valores de $u$ tendem a zero, o que poderá ser percebido pela análise da representação gráfica de $u$. O segundo objetivo é possibilitar uma primeira discussão, de forma bastante intuitiva, a respeito do conceito de limite no infinito de uma função, noção que será discutida nas aulas subsequentes a essa revisita às noções relacionadas às funções.

O foco da questão 12 é possibilitar que o estudante perceba, em primeiro lugar, de maneira intuitiva e sem necessariamente mencionar esses termos, que a função $u$ é limitada superiormente pela função $f$ e inferiormente pela função $-f$. A partir desta percepção, o professor poderá explorar ideias iniciais relacionadas ao Teorema do Confronto, outro objeto matemático que será melhor explorado nas aulas seguintes. Além disso, ao solicitar que o estudante busque valores de $t$ para os quais $u(t)=f(t)$ e aqueles para os quais $u(t)=$ $-f(t)$, o professor poderá explorar, além da noção de intersecções de representações gráficas de funções, a resolução de equações trigonométricas em $\mathbb{R}$, uma vez que:

$$
\begin{gathered}
u(t)=f(t) \Leftrightarrow \cos (10 t-0,05)=1 \\
\text { e } u(t)=-f(t) \Leftrightarrow \cos (10 t-0,05)=-1 .
\end{gathered}
$$

$\mathrm{Na}$ questão 13, o objetivo é possibilitar ao estudante que estabeleça relações entre a ideia matemática de estudar o sinal de uma função, no caso $u$, e o significado físico deste estudo no contexto do problema que está sendo considerado: valores de $u$ menores do que zero indicam que naqueles instantes em que eles são assumidos, o pórtico está se deslocando horizontalmente para a esquerda; valores de $u$ maiores do que zero indicam que naqueles instantes em que eles são assumidos, o pórtico está se deslocando horizontalmente para a direita e valores de $u$ iguais a zero indicam que nos instantes $t$ em que eles ocorrem, o pórtico está na posição inicial.

O objetivo da questão 14 é que o estudante perceba que, para identificar os ciclos da função $u$, precisará determinar os pontos de intersecção entre as representações gráficas das funções, dadas algebricamente por:

$$
f(t)=0,10 e^{-0,50 t} \text { e } u(t)=e^{-0,50 t}[0,10 \cos (10 t-0,05)]
$$

A distância entre as abscissas de dois pontos consecutivos de intersecção destas representações gráficas será o pseudoperíodo de $u$. As amplitudes da função $u$ serão os valores das ordenadas dos pontos de intersecção entre as representações gráficas de $f$ e $u$. Finalmente, na questão 15 , pretende-se que o estudante mobilize os conhecimentos construídos por meio das questões anteriores para que possa efetivamente dar a resposta à 
questão principal do problema. De posse desses conhecimentos, responder ao que é pedido exige do graduando, se ele optar por uma resolução algébrica, resolver a equação:

$$
\begin{aligned}
u(t) & =20 \% \cdot u_{0}=0,2 \cdot u_{0}=0,2 \cdot 0,1=0,02 \Leftrightarrow \\
& \Leftrightarrow e^{-0,50 t}[0,10 \cos (10 t-0,05)]=0,02
\end{aligned}
$$

Neste momento, o estudante deve recordar, a partir das análises que realizou ao responder as questões 12 e 14 , que no instante $t$ em que a amplitude é $20 \%$ da inicial, $\cos (10 t-0,05)=$ 1. Assim, para obter o valor de $t$ para o qual $u(t)=0,02$ basta resolver a equação exponencial: $0,10 e^{-0,50 t}=0,02 \Leftrightarrow t=3,22$. Logo, a amplitude será $20 \%$ da inicial 3,22 segundos depois de a estrutura começar a vibrar. Com a análise realizada ao resolver questão 14 , o estudante perceberá que cada ciclo tem duração de 0,63 segundo. Portanto, o número de ciclos para que a amplitude seja $20 \%$ da inicial será dado por: $\frac{3,22}{0,63}=5,11 \cong 5$, ou seja, são necessários 5 ciclos para que a amplitude se reduza na proporção desejada.

Caso opte por uma resolução gráfica, basta que o graduando determine a abscissa do ponto de intersecção da reta $y=0,02$ com a representação gráfica de $f(t)=0,10 e^{-0,50 t}$, que será $t=3,22$ (observando, mais uma vez que, no instante $t$ procurado basta considerar essa função exponencial uma vez que $\cos (10 t-0,05)=1$ ) e em seguida utilize o fato de que cada ciclo tem duração de 0,63 segundo e determine o que é solicitado.

\section{CONSIDERAÇÕES ACERCA DE COMPETÊNCIAS QUE PODEM SER MOBILIZADAS OU CONSTRUIIDAS AO LONGO DA INTERVENÇÃO}

No que tange à formação do engenheiro, destacamos as seguintes competências gerais indicadas nas DCN (BRASIL, 2019) como desejadas ao egresso: I - formular e conceber soluções desejáveis de engenharia, analisando e compreendendo os usuários dessas soluções e seu contexto, II - analisar e compreender os fenômenos físicos e químicos por meio de modelos simbólicos, físicos e outros, verificados e validados por experimentação; III conceber, projetar e analisar sistemas, produtos (bens e serviços), componentes ou processos; IV - implantar, supervisionar e controlar as soluções de Engenharia; V - comunicar-se eficazmente nas formas escrita, oral e gráfica.

Entre as oito competências gerais elencadas nas DCN, demos destaque para as cinco anteriormente mencionadas por acreditarmos que estas, especialmente, podem ser potencializadas pela área da Matemática, quando recorre-se a uma abordagem desta ciência fundamentada por situações contextualizadas juntamente com estratégias didáticas que mobilizem as oito competências matemáticas apontadas por Niss (2003), a saber: (i) Pensar matematicamente; (ii) Raciocinar matematicamente; (iii) Apresentar e solucionar problemas matemáticos; (iv) Modelar matematicamente; (v) Representar entes matemáticos; (vi) Manipular símbolos matemáticos e trabalhar com o formalismo matemático; (vii) Comunicarse em, com e sobre a Matemática; (viii) Fazer uso de instrumentos e ferramentas de forma eficiente e reflexiva. Essas competências possuem certo grau de imbricação e estão relacionadas aos processos mentais e físicos, atividades e comportamentos, evidenciando aquilo que o indivíduo pode fazer tendo por base o conhecimento matemático (NISS, 2003).

Camarena (2010, p. 8 - tradução nossa) nos ajuda, de certa forma, a pontuar enlaces entre as competências gerais postuladas pelas DCN (BRASIL, 2019) e as competências matemáticas apontadas por Niss (2003), quando salienta que na Engenharia, a Matemática desempenha os seguintes papéis: (i) Permite prever comportamentos; (ii) Permite melhor manejo da linguagem da Engenharia; (iii) Auxilia a otimizar projetos e recursos; (iv) Favorece à minimização de erros; (v) Permite realizar cálculos teóricos ao invés de cálculos práticos e com isso economizar tempo e recursos; (vi) Proporciona maior precisão à análise de um 
problema; (vii) Permite o desenvolvimento de ordens lógicas de ação; (viii) Possibilita o desenvolvimento de um espírito científico; (ix) Favorece o ser analítico e crítico; (x) Permite o desenvolvimento de raciocínios importantes para a Engenharia e para a vida profissional do engenheiro.

Acreditamos que a intervenção didática apresentada tem potencial para, na formação do futuro engenheiro, proporcionar a mobilização ou o desenvolvimento tanto daquelas competências gerais explicitadas nas novas DCN e por nós destacadas anteriormente, quanto daquelas específicas da área da Matemática sinalizadas por Niss (2003).

A experiência em sala de aula ministrando disciplinas da área da Matemática combinada com os estudos, como os realizados por Camarena $(2010,2013)$, permite aferir que um ensino contextualizado da Matemática nos cursos de Engenharia tem implicação direta na motivação do aluno em estudar os conceitos desta ciência, além de contribuir para que os estudantes construam os conhecimentos matemáticos de forma não fracionada, com mais solidez e, principalmente, abre possibilidades para aprendizagens significativas (AUSUBEL; NOVAK; HANESIAN, 1990).

Por fim, é relevante pontuar a importância da integração entre os professores das áreas básicas e específicas da Engenharia para a compreensão do potencial do problema dos pórticos para sua abordagem nas disciplinas de Cálculo Diferencial e Integral. No diálogo que resultou no desenvolvimento da intervenção proposta, duas engenheiras apresentaram aos matemáticos a solução do problema e estes se detiveram a refletir a respeito dos conceitos matemáticos empregados; das adaptações necessárias para utilização do problema em aula; dos objetivos da proposição do problema nas disciplinas básicas; das eventuais divergências de linguagem e de notação; entre outros. À medida em que os matemáticos buscavam compreender a solução do problema, dialogavam com as engenheiras a respeito de pontos que estavam ainda "nebulosos" no que se referia aos conteúdos do contexto da Engenharia Civil mobilizados. Pouco a pouco as professoras engenheiras compreendiam de maneira mais efetiva como determinados conceitos da Matemática estavam sendo mobilizados na situação, ainda que de maneira implícita, e puderam perceber que a Matemática é mais do que uma ferramenta empregada pelos engenheiros; essa ciência, em muitos casos, embasa as teorias subjacentes às diferentes situações específicas da Engenharia, bem como seus modelos.

Em síntese, o diálogo realizado entre docentes das áreas básicas e específicas, revelouse profícuo especialmente porque as engenheiras auxiliaram os matemáticos a compreender o problema sob o ponto de vista da Engenharia e estes, por sua vez, contribuíram para que elas percebessem a conexão dos conteúdos que lecionam com os que os graduandos estudam nas disciplinas das áreas básicas, para oportunamente evidenciá-los aos estudantes.

\section{Agradecimentos}

Agradecemos às engenheiras civis Karina Bradaschia Rocha e Paula Meirelles Bolelli que atuaram de forma colaborativa no trabalho de desenvolvimento da situação contextualizada que deu origem à intervenção apresentada neste trabalho.

\section{REFERÊNCIAS}

AUSUBEL, D. P., NOVAK, J. D.; HANESIAN, H. Psicología educativa, un punto de vista cognoscitivo. México: Editorial Trillas, 1990.

BIANCHINI, B. L. et al. Competências matemáticas: perspectivas da SEFI e da MCC. Educação Matemática Pesquisa, São Paulo, v.19, n.1, p. 49-79, 2017.DOI:

http://dx.doi.org/10.23925/1983-3156.2017v19i1p49-79. 
BRASIL. Ministério da Educação. Resolução CNE/CES n. 2/2019, de 23 de abril de 2019. Institui as Diretrizes Curriculares Nacionais do Curso de Graduação em Engenharia, 2019b. Disponível em: < http://www.in.gov.br/web/dou/-/resolu\%C3\%87\%C3\%83o-n\%C2\%BA-2de-24-de-abril-de-2019-85344528>. Acesso em: 06 mai. 2020.

CAMARENA, P. A treinta años de la teoría educativa "Matemática en el contexto de las ciencias", Revista Innovación Educativa, Vol. 13, Núm. 62, p.17-44, 2013.

P. Aportaciones de Investigación al Aprendizaje y Enseñanza de la Matemática en Ingeniería, 2010. Disponível em:

<http://www.ai.org.mx/ai/archivos/ingresos/camarenagallardo/dra._patricia_camarena_gallard o.pdf $>$ - Acesso em 02 de jun. de 2020.

LIMA, G. L. Abordagem contextualizada e compreensão relacional: em busca de uma identidade para o curso inicial de Cálculo. In: Comité Interamericano de Educación Matemática (2015). Educación Matemática en las Américas: 2015. Volumen 10: Álgebra y Cálculo. Editores: Patrick (Rick) Scott y Ángel Ruíz. República Dominicana.

NISS, M. Mathematical Competencies and the Learning of Mathematics: The Danish KOM project. In: GAGATSIS, A. e PAPASTRAVIDIS, S. (Eds.). $3^{\circ}$ Mediterranean Conference on Mathematics Education 2003. Atenas - Grécia: Hellenic Mathematical Society and Cyprus Mathematical Society, pp.115-124, 2003.

\title{
THE GANTRIES' PROBLEM: A DIDACTIC INTERVENTION BUILT FOR A DIFFERENTIAL AND INTEGRAL CALCULUS COURSE
}

\begin{abstract}
The goal of this article, one of the fruits of the demands arising from reflections that occurred in the meetings of 2017 to 2019 of the Working Group Basic Sciences and Mathematics in Engineering, linked to the Brazilian Association of Education in Engineering, is to present a didactic intervention, developed, for an initial course of Differential and Integral Calculus, from a classic problem in Civil Engineering - the gantries' problem through dialogue between teachers in the area of Mathematics and teachers who teach specific or professional courses in the Civil Engineering programs. We explain the problem that contextualizes the proposed intervention, planned to be developed in 12 hours/class (approximately 3 weeks with 4 hours/class in each one), and then we detail what was designed to be developed in each one classes contemplating such intervention. We believe that the proposal presented has the potential, in the training of the future engineer, to provide for the development or mobilization of both some general competences explained in the new National Curricular Guidelines for Undergraduate Engineering courses, as well as specific competences in the area of Mathematics postulated by Mogens Niss.
\end{abstract}

Keywords: Civil Engineering, Gantries' Problem, Differential and Integral Calculus, Didactic Intervention, Competences. 